LA INFLUENCIA DE

LA POSTMODERNIDAD EN

ORGULLO Y PREJUICIO

(1813): PERSIGUIENDO A

JANE AUSTEN (2008),

\section{DAN ZEFF}

\section{Irene Romero González}

\section{Doctora}

Universidad Complutense de Madrid. Ciudad Universitaria, 28040 Madrid - Email: ireneromerogonzalez@gmail.com

\section{Resumen}

Las novelas de Jane Austen siguen inspirando nuevos relatos cinematográficos pues éstos toman sus historias o sus personajes y los adaptan a contextos culturales y axiológicos actuales de gran diversidad. Es el caso de la novela Orgullo y Prejuicio de la que se han realizado numerosas adaptaciones clásicas, así como que ha sido objeto de referencia en películas, en trabajos literarios y de adaptaciones postmodernas. Uno de estos trabajos postmodernos es Persiguiendo a Jane Austen, una serie inglesa que nos adentra en el mundo de la Regencia a través de las vivencias de una mujer contemporánea que descubre una puerta que comunica ambos mundos y que le permite vivir la
Palabras clave

Postmodernidad, Orgullo y

Prejuicio, Persiguiendo a Jane

Austen, cine, literatura,

anacronismos

Key Words

Postmodernism, Pride and

Prejudice, Lost in Austen, cinema, literature, anachronism

\section{Abstract}

The novels of Jane Austen continue to inspire new film stories as they take their stories or their characters and adapt them to cultural and axiological current contexts of great diversity. This is the case of the novel Pride and Prejudice which has been widely adapted and has been referenced in films, literary works and postmodern adaptations. One such work is the postmodern Lost in Austen, a British serie that takes us into the world of the Regency through the experiences of a contemporary woman who discovers a door connecting the two worlds and lets her lives the story of Austen under the Elizabeth Bennet skin. Throughout this paper we will perform a comparative analysis between this adaptation and its postmodern literary reference to see how the ideas of

Postmodernism influence on a classic nineteenth-century literature. It is a trend that helps us to rethink a period of history and to

reinterpret the context of the original novel to a different and original approach. 
historia de Austen bajo la piel de Elizabeth Bennet. A lo largo de este trabajo se realizará un análisis comparado entre esta adaptación postmoderna y su referente literario, para comprobar cómo influyen las ideas de la Postmodernidad en un clásico de la literatura decimonónica, tendencia que nos ayuda a replantearnos un periodo de la historia y a reinterpretar el contexto de la novela original a uno distinto y original.

\section{Introducción}

Es una verdad universalmente reconocida que Jane Austen es una fuente inagotable de estudio y su influencia va más allá de la literatura. A través de las adaptaciones fílmicas de su obra, conocemos las historias que esta autora creó en el siglo XIX y, en numerosas ocasiones, sus novelas continúan inspirando relatos cinematográficos en los que se adaptan sus historias a contextos culturales y axiológicos actuales de gran diversidad.

La novela Orgullo y Prejuicio fue creada en 1813 y desde la aparición del cine se han realizado once adaptaciones clásicas (aquellas que son fieles a la esencia que la autora le confirió en su creación) sobre la historia protagonizada por Fiztwilliam Darcy y Elizabeth Bennet; pero, también existen adaptaciones teatrales ${ }^{\mathrm{i}}$, referencias en dibujos animadosii ${ }^{\mathrm{ii}}$ en seriesiii y en películas $^{\mathrm{iv}}$, trabajos literarios ${ }^{\mathrm{v}}$ y adaptaciones postmodernas ${ }^{\mathrm{vi}}$, que son las que se abordarán en este trabajo.

Las adaptaciones postmodernas nos ayudan a replantearnos el período de- cimonónico de Austen "como etapa de la historia y como ámbito cultural y literario parec[iendo] ser una respuesta a la nueva concepción postmoderna del discurso histórico" (Pérez Ríu, 2000:95). De esta manera, este nuevo enfoque permite mayor libertad para interpretar el contexto donde se inscribió la novela original, siendo adaptada a una nueva concepción cultural e histórica. Estas adaptaciones postmodernas son menos realistas y se alejan de la fidelidad respecto a la novela, pero reconocen la distancia histórica que les separa del original. La interpretación contemporánea que se hace de los personajes de la novela redefine sus caracteres y sus comportamientos.

Esta falta de realismo y fidelidad se debe a que el discurso postmoderno no se toma en serio la narración literaria y recurre al humor, a la parodia, al absurdo o a la intertextualidad para relatar la historia. La Postmodernidad juega con un referente previo existente, como es la novela original, reelaborando ese texto que ya existe, confiriéndole un nuevo significado y ten- 
diendo, por tanto, hacia la infidelidad con respecto al original. El texto postmoderno adaptado no busca la integración de la historia, la síntesis de la misma o la totalidad de lo narrado; no busca fidelidad ni coherencia, sino una interacción y una mezcla de los dos textos.

La Postmodernidad cuestiona todos los principios esenciales de la Ilustración: las jerarquías del conocimiento, del gusto y de la opinión, y se sustituye el libro impreso por la pantalla de televisión, pasándose de la palabra a la imagen. Aunque lo postmoderno surge como un rechazo a lo moderno, no implica, sin embargo, un rechazo a todo lo heredado de la modernidad. A pesar de que la postmodernidad se aísle de su pasado inmediato ha establecido unos mecanismos que favorecen una lectura crítica sobre los mode- los canónicos, hegemónicos y dominantes propios de la modernidad. Así, el postmodernismo artístico rechaza el elitismo burgués, la presuntuosidad del modernismo, el realismo, la mímesis, las formas narrativas lineales, combinando formas culturales de distintas clases sociales en una estética plural y popular. Este movimiento aboga por la hibridación de formas, de géneros, de estilos culturales o de periodos temporales, además de por la descontextualizazión y recontextualización de estilos arquitectónicos, artísticos y literarios ${ }^{\mathrm{vii}}$. En este trabajo se analizará la adaptación postmoderna Persiguiendo a Jane Austen (2008) que toma como base la historia y los personajes de la novela Orgullo y Prejuicio de Austen y los adapta a los valores culturales, sociales y políticos de nuestra sociedad contemporánea.

\section{Objetivos}

La literatura ha sido, desde siempre, una vía de comunicación de gran importancia porque a través de ella se transmiten historias ficticias o reales con las que se trata de influir en el lector. Al interactuar con otros discursos o tendencias, como el cine o la Postmodernidad, nos permite descubrir nuevas interpretaciones del texto literario, además de inspirar la creación de nuevos textos cinematográficos. Por esta razón, el objetivo principal del presente artículo es analizar cómo influye la Postmodernidad en las obras de grandes escritores, como las de Jane Austen, para la creación de un nuevo discurso audiovisual autónomo e irreverente, que no busca la fidelidad con respecto al texto original sino una interacción y una mezcla de los dos discursos narrativos, adecuando los carac- 
teres de los personajes a una nueva realidad cultural, social e histórica.

\section{Metodología}

Desde un punto de vista metodológico, el presente artículo comenzaría con la lectura de la novela de Jane Austen, Orgullo y Prejuicio (1813) y del visionado de la serie Persiguiendo a Jane Austen (2008) para analizar los elementos comunes y dispares que surgen cuando se adapta un texto literario al medio audiovisual. Además de acotar el objeto de estudio: la influencia de la Postmodernidad en la reinterpretación del clásico de Austen. Para comprender dicha influencia, se analiza el con- cepto de Postmodernidad para realizar el análisis comparado con la novela teniendo en cuenta la distancia histórica existente y su creación bajo estas ideas. Este trabajo no se centra en los elementos dispares, pues desde el inicio soy consciente de que esta versión sólo toma como modelo la historia y los personajes, por lo que se analizan aquellos aspectos que la hacen similar a la novela y, por tanto, mantienen una conexión con la obra de Austen.

\section{Aproximación al concepto de Postmodernidad}

La Postmodernidad es un movimiento cuya definición es bastante ambigua y difícil de concretar pues abarca ámbitos muy variados y dispares. Así, Postmodernidad, en su primera aproximación, fue definida en términos arquitectónicos aunque adaptada, también, a otros medios artísticos como la literatura y las artes visuales, y a distintas corrientes filosóficas y sociales. No se puede considerar a la Postmodernidad, exclusivamente, como un acontecimiento cultural, pues también está relacionada con un nuevo tipo de sociedad, la denominada postindustrial, de consumo o de la información.

François Lyotard (1987) en su introducción a La Condición Postmoderna especifica que el término de Postmodernidad es una "condición", un estado caracterizado por el fin o la inoperancia de las ideologías modernas. Afirma, además, que este concepto "[d]esigna el estado de la cultura después de las transformaciones que han afectado a las reglas de juego de la ciencia, de la literatura y de las artes a partir del siglo XIX” (1987:4). Albre- 
cht Wellmer (1993), por su parte, acude a la raíz de la palabra («post») para indicarnos que Postmodernidad forma parte

de una red de conceptos y formas de pensamiento «postísticas» sociedad post-industrial, postestructuralismo, post-empirismo, post-racionalismo - en los que al parecer trata de articularse la conciencia de hallarse en el umbral de una época cuyos contornos son aún confusos, poco claros y ambiguos, pero cuya experiencia central sin embargo - la muerte de la razónparece apuntar al final definitivo de un proyecto histórico: el proyecto de la modernidad, el proyecto de la Ilustración europea, o incluso, por último, el proyecto de la civilización greco-occidental. (1993:51)

Añade también, que dependiendo de la perspectiva desde la que se estudie la Postmodernidad, conferirá al concepto un significado distinto. Ante la falta de un marco teórico en el que definir el concepto de postmodernidad, Lyotard enfoca su estudio hacia el saber científico y su evolución en la sociedad. Para él, el saber es distinto dependiendo de la sociedad en la que se encuentre; así, el saber en las sociedades con una cultura postindustrial difiere de aquel por el que se rigen las culturas postmodernas.
Alison Assiter (1996) nombra a Andreas Huyssen como un ejemplo de las distintas definiciones que abarca el término. Para este autor existen cuatro características esenciales de la fase inicial del postmodernismo, acaecido en la década de 1960:

First $[\ldots]$ postmodernism was characterised by 'a temporal imagination which displayed a powerful sense of the future and of new frontiers, of rupture and discontinuity, of crisis and generational conflict [...]. Second, the early phase of postmodernism included an iconoclastic attack on what Peter Burger has tried to capture theoretically as 'institution art'....] Against this, postmodernists advocated pop, psychedelic art, acid rock, alternative and street theatre, focusing on mass culture as against 'high' art.

Huyssen's third characteristic [...] is a 'technological optimism': Mcluhan's cybernetic and media eschatology 'all combined easily with euphoric visions of a postindustrial society'. Finally, postmodernism, Huyssen suggests, heralded a wider reaction against 'modern' society: 'postmodernism harboured the promise of a "post male", "post white", "post humanist" and "post puritan" world'. (1996:2) 
Aunque estas características se hayan producido en la década de los 60 , actualmente se mantienen, pues la sociedad ha avanzado en los términos tecnológicos mencionados por Huyssen y se ha expandido a otros continentes y a otras áreas de actuación más específicas.

Fredric Jameson (1991) para definir este movimiento enumera una serie de características que abarcan un amplio abanico de discursos (artístico, cultural, literario y filosófico):

- el fin de la ideología, del arte o de las ciencias sociales,

- la "crisis" del leninismo, de la socialdemocracia o del estado de bienestar,

- debilitamiento o extinción del movimiento modernista, a pesar de los pequeños impulsos producidos con el expresionismo abstracto en pintura o el existencialismo en filosofía,

- la aparición del pop-art con Andy Warhol, del fotorrealismo y el "nuevo expresionismo; del punk y el rock new wave, del cine de Godard, o del cine experimental y el comercial (1991:15),

- desaparición o disminución de los afectos, los sentimientos, las emociones y la subjetividad de los indi- viduos por la liberalización de la sociedad (1991:27; 32),

- y, por el pasado que pierde importancia para el individuo el cual sólo quiere vivir el presente (1991:3546).

La Postmodernidad cuestiona todos los principios esenciales de la Ilustración y del modernismo. De esta manera, se quiebran las jerarquías del conocimiento, del gusto y la opinión. Se impone una visión "débil" de la ciencia y la autoridad pierde su lugar privilegiado. Se sustituye el libro impreso por la pantalla de televisión, se pasa de la palabra a la imagen, del logocentrismo al iconocentrismo. El postmodernismo se ha producido en tres movimientos diferentes definidos por Alex Callini$\cos (1993)$ :

$[\mathrm{P}]$ rimero incluye algunos cambios ocurridos en las artes [...] en particular, la reacción en contra del Estilo Internacional en arquitectura, vinculada con nombres tales como Robert Venturi y James Sterling [...]. Este rechazo del funcionalismo y la austeridad, [...] halló aparentes paralelos en [...] el regreso al arte figurativo en pintura, por ejemplo, y la narrativa de escritores como Thomas Pynchon y Umberto Eco.

En segundo lugar, [...] un grupo de teóricos franceses [...] llegaron a 
ser conocidos durante los años setenta en el mundo de habla inglesa bajo el rótulo de "postestructuralistas": [...] Gilles Deleuze, Jacques Derrida y Michel Foucault. [...] [T]odos ellos enfatizaron el carácter fragmentario, heterogéneo $y$ plural de la realidad, negaron al pensamiento humano la capacidad de alcanzar una explicación objetiva de esa realidad y redujeron al portador de este pensamiento, el sujeto, a un incoherente revoltijo de impulsos y deseos sub y transindividuales.

$[\ldots][E] n$ tercer lugar, el arte y la filosofía parecían reflejar [...] cambios ocurridos en el mundo social. [...] [E]l mundo desarrollado se encuentra en una etapa de transición de una economía basada en la producción industrial masiva hacia una economía en donde la investigación teórica sistemática se constituye en el motor del crecimiento, una transformación de incalculables consecuencias sociales, políticas y culturales. (1989:10)

Para Ágnes Heller y Ferenc Fehér, la Postmodernidad es una etapa «parasitaria» del movimiento modernista pues "vive y se alimenta de sus logros y dilemas", a pesar de que el individuo postmoderno vive por y para el presente (Heller y Fehér, 1989:161). En cam- bio, otros autores consideran que estamos ante un periodo artístico ecléctico. Es decir, ante un nuevo movimiento que mezcla distintas concepciones filosóficas, ideas y valoraciones de distintas escuelas, en forma de pastiche, pues con la mezcla de esos pensamientos se trata de dar la impresión de ser una creación independiente $\mathrm{y}$ novedosa $a^{\text {viii }}$.

\subsection{La Postmodernidad $y$ otros movimientos sociales $y$ artísticos}

Postmodernidad no es sólo un término empleado en distintas áreas artísticas, filosóficas o literarias, sino que, además, ampara otras teorías con las que interactúa como es el estructuralismo, el deconstructivismo o el feminismo a los que denomina: postestructuralismo, postdeconstructivismo o postfeminismo. En cuanto al ámbito cinematográfico, también surge una crítica cinematográfica postmoderna y feminista. El género cinematográfico femenino, por excelencia, es el romance y el drama. Los romances han sido desde hace mucho tiempo el tema principal de mujeres novelistas como Maria Edgeworth. Sin embargo, desde su inicio, estas escritoras temieron que, a través de sus novelas, inculcaran valores y actitudes que provocasen un descontento en los matrimonios de la época. 
Por eso muchas de estas novelas pueden ser leídas como una crítica al romance (Modleski, 2008:XXIV). A través de estas historias se muestra a las mujeres la existencia de un hombre perfecto y las insta a la búsqueda de una vida feliz junto a él. Con la influencia del feminismo y la postmodernidad, estas historias decimonónicas de apariencia tan perfecta tienden a desaparecer, pues las historias actuales presentan relaciones sentimentales que, a priori, ni son tan sencillas ni tan bonitas. En la mayoría de los casos, las mujeres viven en circunstancias de opresión o son tan feministas que buscan su independencia antes de comprometerse; y sólo alcanzan este compromiso cuando ya han satisfecho sus aspiraciones laborales y personales. Tania Modleski (2008) afirma que la literatura del siglo dieciocho y diecinueve ha influido en la vida contemporánea hasta ser la responsable de muchos de los males de la cultura de masas, además de influir en las películas domésticas (aquellas en las que se muestra y tratan de glorificar la vida doméstica de las mujeres) de los años 1930 y 1940 (2008:5-14). Las mujeres que se representan en estas películas no dejan de ser un reflejo de la sociedad en la que se inscriben, por lo que en muchas de ellas se reconocerán las desigualdades a las que está sometida la mujer en la vida real. Con la apari- ción del feminismo postmoderno, las películas no sólo cambian los roles preestablecidos que encarnan las mujeres de principios del siglo $\mathrm{XX}$, sino, también, el público al cuál van dirigidas: las mujeres jóvenes, que son quienes representan los nuevos cambios sociales y valores de independencia femenina y de igualdad.

Para Roberta Garrett, el cine postmoderno ha perdido de vista el referente histórico y el pasado "representa un surtido de significantes vacíos" (Garrett, 1995:89). Jameson añade que es inquietante que la sociedad postmoderna se aleje del pasado pues es "un síntoma alarmante y patológico de una sociedad que se ha sido incapaz de realizar transacciones entre el tiempo y la historia" (1991:117).

Es en este contexto donde se reinterpretan los grandes clásicos de la literatura, como es el caso de Orgullo y Prejuicio, que ha sido adaptado a una nueva realidad social, histórica y cultural que nada tiene que ver con la de su concepción. La adaptación que se va a analizar en este trabajo, Persiguiendo a Jane Austen, transforma el lenguaje propio de la novela original en un lenguaje que cristaliza un universo de visiones y valores radicalmente distintos. Esta adaptación presenta una vuelta de tuerca a la historia de amor entre Darcy y Elizabeth. 


\section{Persiguiendo a Jane Austen (2008), Dan Zeff}

Persiguiendo a Jane Austen (cuyo título original es Lost in Austen) es una miniserie británica de cuatro capítulos que fue realizada en 2008 por la cadena televisiva ITV. Es una peculiar adaptación, casi a modo de parodia, de la novela Orgullo y Prejuicio. La protagonista, Amanda Price, que vive en el Londres contemporáneo, harta de su monótona vida y de su novio, se refugia en su novela favorita, Orgullo y Prejuicio, hasta que un día se encuentra en el baño a Elizabeth Bennet quien afirma que hay una puerta que comunica las dos casas. Cuando Amanda decide comprobar la veracidad de sus palabras, se queda atrapada en el mundo de Elizabeth, mientras ésta se queda en el Londres actual. A partir de entonces la historia que creó Austen no sucede tal como ella la escribió, sino que se desarrolla de manera diferente, hasta el punto de que Jane Austen podría revolverse "en su tumba como si fuera un gato en una secadora" (Persiguiendo a Jane Austen, Cap.4, 00:12:00).

Esta serie fue emitida por primera vez en la cadena ITV-1 en septiembre de 2008 con una buena recepción por parte del público y de la crítica. El primer capítulo consiguió una cuota de share de $17,6 \%$ equivalente a 4.185.000 espectadores. Sarah Dempster, en su blog del periódico The Guardian, analizó los cuatro capítulos de la serie afirmando que no importa que uno se sienta perdido y le parezca todo improbable, porque "[s]o perfectly drawn is the world that begins to unfurl - and so sincere and endearing is Guy Andrews' script - that suspension of disbelief becomes part of the fun. It's a fantasy. A fairy tale" (Dempster, 2008).

Tim Teeman en The Times escribió que esta versión de Jane Austen es muy divertida e inteligente y que el hecho de que Amanda se introduzca en el mundo de Elizabeth Benne "[i]t is a culture-clashing, time-clashing Walnut Whip of frothy nonsense with the intriguing proposition that Amanda may be able to change the outcome of her fictional touchstone. But what's Elizabeth getting up to in Hammersmith?" (Teeman, 2008).

A pesar del buen comienzo, los posteriores capítulos de Lost in Austen no alcanzaron la cuota inicial de audiencia en la cadena británica ITV, por lo que tras el último capítulo emitido, alcanzó un promedio general de cuota 
del $14,1 \%$ que supone unos 3.200 .000 espectadores (Wilkes, 2008). En el artículo "'Completely without sense': Lost in Austen" (2008), Laura Kaplan analiza el público objetivo al que iba dirigida la serie alegando que existen tres segmentos de público distintos:

[ $t$ ] he primary audience is the twenty-somethings familiar with magical doors, time-travel tales, and cultural absurdities. These Generation$Y$ viewers recognize the classic Elizabeth and Darcy love story from assigned readings in schools and universities, and from films and television. They are au courant with text messaging, urban slang, high street fashion and accessories, and the rituals and problems of twenty-first-century romantic relationships. The second target audience, a more general group that incorporates the first, primarily younger, segment, is made up of viewers familiar with (and likely enamored of) Colin Firth's wet shirt scene in Andrew Davies's 1995 series, with the Bridget Jones books and films, and with Elizabeth Bennet's glamorous incarnation as Keira Knightley in Joe Wright's 2005 film Pride \& Prejudice. These viewers may be slightly older than twenty-anything; it may have been awhile since they have read P\&P or any of Austen's novels; and they are bound to be baffled by some of the contemporary women's magazine expressions, silly jokes and contemporary references, and the occasionally rude vocabulary. The third segment of the target audience is the Janeites, of course, and they may well be the viewers most inclined to resist this sort of over-thetop pastiche. If Janeites are too fussy about textual fidelity, however, they risk missing something they will actually end up appreciating more than the other demographics. (2008:244)

Persiguiendo a Jane Austen es una historia postmoderna que toma como base un texto literario (ficción) para adecuarlo a la realidad contemporánea, adquiriendo de la novela aquellos referentes que más la caracterizan para reelaborarlos y adaptarlos a un contexto y a un soporte totalmente diferentes. Por esta razón, no busca la fidelidad con el texto literario, sino una nueva interpretación de la historia. Persiguiendo a Jane Austen transforma el deseo de muchas Janeites ${ }^{\text {ix }}$ de sumergirse y formar parte del mundo de las novelas de Austen, y lo convierte en una realidad (representada). El universo de Orgullo y Prejuicio les ayuda a apuntalar sus vidas faltas de amor romántico, cortesía, códigos de comportamiento y maneras, siendo Amanda la 
persona que representa a este grupo, pues como ella afirma:

No estoy enganchada a Darcy. No estoy todo el día en casa delante de la tele viendo a Colin Firth en pantalones de montar, ¿vale? Me gus$\tan . .$. las historias de amor. Me gusta Elizabeth. Me encantan... sus modales y... su lenguaje y... su cortesía... Ese mundo se ha convertido en parte de mi mundo. (Cap.1, 00:05:55 - 00:06:21)

Kaplan asegura que Orgullo y Prejuicio se ha convertido en una especie de manual de cortesía para esta generación hasta el punto "that it is not only Amanda who uses the novel as a guide to life's values. Young women want 'gentlemen' who will court them and pay for dinner, even after the first date" (2010).

Por esta razón, a lo largo de esta serie no sólo vemos elementos propios de la novela de Austen, sino que observamos referencias de otras novelas y otras adaptaciones cinematográficas clásicas a la obra literaria de Austen que fueron el referente para las Janeites. Sin embargo, para darse cuenta de estas alusiones no sólo es necesario conocer previamente todas las versiones que se han hecho de Orgullo y Prejuicio, sino también conocer el resto de la obra de la autora. Así, encontramos menciones a la serie reali- zada por la BBC en 1995 y a la película de Joe Wright de 2005 que aportan "un curioso momento postmoderno" (Cap.3, 00:29:58).

Como en otras adaptaciones postmodernas que se han hecho de Orgullo y Prejuicio, el primer capítulo empieza con la famosa frase de Austen "Es una verdad universalmente reconocida...", pero a diferencia de otras versiones, en esta ocasión el tema central no es el matrimonio, sino la evasión de la realidad y lo que implica. De esta manera la trama principal es la capacidad que tiene Amanda de abstraerse de la realidad (representada) y adentrarse en un mundo ficticio:

Es un hecho generalmente aceptado que todos nos queremos evadir. Yo... siempre me evado con mi libro favorito... Orgullo y Prejuicio. Lo he leído tantas veces que las palabras resuenan por sí solas en mi mente y... es como si se abriera una ventana. Parece como si realmente estuviera allí. Lo conozco $\tan . .$. a fondo que puedo ver ese mundo... Puedo tocarlo... Puedo ver a Darcy. (Cap.1, 00:00:04 00:00:45).

El uso de esta frase al inicio (o en el transcurso de la película, como es el caso de El diario de Bridget Jones) establece la trama principal de la historia y confiere a la versión el sello pro- 
pio de la novela reafirmándose como heredera de la misma. No es la única cita propia de la novela, sino que a lo largo de los cuatro episodios aparecen algunas más. Sin embargo, con la constante interferencia de Amanda en la evolución de la historia y en las acciones, estas referencias no se suceden de la misma manera que Austen las escribió, sino que han cambiado y, con ello, los diálogos que se producen en ellas. A lo largo de esta serie, se seleccionan los fragmentos más destacados recomponiéndolos en nuevas acciones y funciones, obteniéndose, así, un resultado totalmente diferente.

En Persiguiendo a Jane Austen existen dos realidades contrapuestas, dos códigos de comportamientos sociales diferentes, dos culturas opuestas y dos lenguas distintas. Aunque sean la misma, algo que se produce en el momento en el que el relato (la historia real-representada) está dentro del relato (historia ficticia): Amanda lee la novela y se mete dentro de ella. Esa irrupción provoca que los personajes de la novela, no sólo se desarrollen siguiendo un guión establecido, sino que, como he apuntado, se desarrollen de manera diferente adaptándose a la nueva realidadficticia y, en ocasiones, atraviesen el marco temporal y espacial que existe entre los dos niveles de realidad. Esta intrusión de Amanda en la historia ficticia conlleva que los acontecimientos ya no se produzcan como Austen los creó sino que cambien y se adapten a la nueva situación. Situación que provoca que Amanda trate de que los hechos se sucedan tal como la autora los concibió. La aparición de Amanda en la era de la Regencia, no sólo determina la incorporación de acciones y situaciones inventadas, sino también la introducción de un bagaje cultural y social intrínseco a la protagonista que interfieren en la correcta consecución de la historia. De esta manera, observamos además anacronismos que son incorporados de manera intencionada por el guionista para provocar situaciones cómicas o malentendidos culturales en los momentos de mayor incomodidad para la protagonista: expresiones verbales, objetos y vestimenta contemporáneos. También, se observa una gran influencia no sólo de otras novelas escritas por Jane Austen sino de otras adaptaciones cinematográficas de Orgullo y Prejuicio producidas con anterioridad. 


\subsection{Acciones \\ $y$ situaciones inventadas con la intrusión de Amanda}

La intrusión de Amanda en la historia ficticia de Austen conlleva que los acontecimientos ya no se produzcan tal y como esta escritora los escribió, sino que cambien y se adapten a la nueva situación. Por más que Amanda trate de que los hechos sucedan como la autora los concibió, la historia no sucede como tal, sino que se desarrolla siguiendo un nuevo guión. Son muchas y variadas estas acciones y situaciones inventadas sin embargo se enumerarán las más importantes e impactantes.

La primera variación con respecto a la novela es el intercambio temporal e histórico de las protagonistas. Elizabeth deja de ser el personaje protagonista de la historia cediéndole el puesto a Amanda, un personaje inventado y ajeno a la historia concebida por Austen. Una vez en Longbourn, Amanda, con su ropa de mujer contemporánea, es presentada a Bingley, quien cae rendido ante el escote que ella luce. En la primera ocasión que él tiene se intenta declarar, pero Amanda le dice que "le gustan las mujeres" y que debe fijar los ojos en otra persona, Jane. Sin embargo, por mucho que trate de intervenir en la relación de Jane y Bingley, éste es convencido por Darcy (como sucede en la novela) y se aleja. Por lo que Jane decide casarse con el Sr. Collins como única solución para conservar la casa de Longbourn como propiedad de la familia Bennet. En la novela, el Sr. Collins se casa con Charlotte Lucas porque ni Elizabeth ni Jane quieren casarse con él. En cambio, en Persiguiendo a Jane Austen, Charlotte decide marchase de misionera a África porque no encuentra marido (Cap.2, 00:40:57). Bingley, deprimido ante la pérdida de Jane, se echa a la bebida y convence a Lydia, una inocente muchacha, para que se vaya con él a Hammersmith y llevar a cabo un "experimento social", pero no sucede nada malo porque han estado toda la noche "filosofando" (Cap.4, 00:12:49 - 00:13:31). Sin embargo, el Sr. Bennet no se cree nada de lo que Bingley dice y le reta, con tan mala suerte de que se da un golpe en la cabeza quedando herido en el suelo (Cap.4, 00:14:03). Otro de los cambios que se suceden es que Caroline Bingley reconoce ante a Amanda que es lesbiana, aunque con otras palabras más propias de su época:

[Caroline] Charles me ha contado su secreto. También es mi secreto... Lanzaré mis garras sobre 
Darcy, y también me casaré con él... porque es justo... y necesario y todo el mundo cuenta con ello, incluido Dios... Pero la cercanía física de los hombres es algo que nunca he buscado... Soportaré una vida con Darcy porque... nuestro género está habituado al sacrificio... Pero la lírica de Safo es la única música que llega a pulsar mi corazón... Aunque todavía no puedo declararme... experimentada en estas artes...Quería que usted lo supiera... Una pequeña confidencia entre hermanas antes de que huya a Hammersmith, pequeña buscona.

$[\ldots]$

[Amanda] [voz en off] ¡Dios mío! ¡Jane Austen se quedaría de piedra si supiera que ha escrito esto!

(Cap.3, 00:40:41 - 00:41:54)

Uno de los cambios más importantes e impactantes es el cambio en el carácter de Wickham. A lo largo de la serie observamos que es un hombre servicial, educado, cortés y ayuda a Amanda siempre que puede: cuando la echan de Longbourn (Cap.3, 00:05:29 00:07:43), o cuando Lydia se fuga con Bingley a Hammersmith (Cap.4, 00:10:02 - 00:12:05). Como consecuencia de este cambio, nos sorprende cuando descubrimos cuáles son los verdaderos motivos por los que Darcy odia a Wickham. En la novela, Darcy asegura que Wickham convenció a su hermana, Georgina, para fugarse con él y quedarse con todo el dinero de su herencia. En cambio, en esta versión no sucede tal como asegura Darcy:

La versión que a usted le han contado no...es lo que sucedió realmente... Mi aya concibió... una pasión... por el Sr. Wickham. Me llevó con ella a un lugar donde podría encontrarse con él... como si fuera por azar. Yo era el pretexto para el plan... Pero yo también me había enamorado de él... Cada vez que mi aya se daba la vuelta, yo me ofrecía a él. Él me llamaba mi dulce niña, su adorable niña, pero niña al fin y al cabo, y acabó rechazándome... De modo que acudí a mi hermano, y le conté que George me había mancillado. (Cap.3, 00:35:02 $-00: 35: 45$ )

A pesar de que Amanda se lamenta, constantemente, que las cosas no ocurren como lo estableció Austen, el final es el esperado y el que aparece, en esencia, en Orgullo y Prejuicio: Jane se casa con Bingley (Cap.4, 00:39:28 - 00:40:08) y Amanda, en representación de Elizabeth, se casa con Darcy (Cap.4, 00:44:13 00:45:10). 


\subsection{Los anacronismos como eje central de la historia}

En Persiguiendo a Jane Austen aparecen gran cantidad de anacronismos que interfieren en la realización de esta historia, pero su incorporación no se debe a un descuido de Guy Andrews, el guionista, sino que son introducidos para provocar situaciones cómicas. Son muchos los que encontramos a lo largo de esta producción inglesa por lo que me voy a centrar en los más significativos. El primero de los anacronismos sucede cuando Elizabeth Bennet aparece en el cuarto de baño de Amanda en el siglo XXI. La protagonista de $\mathrm{Orgu}$ llo y Prejuicio trata de explicarle la situación de la mejor manera:

[Elizabeth] Esto es... absolutamente increíble, pero Srta. Spencer le ruego que dé crédito a mis explicaciones ya que son veraces, aunque soy consciente de que apenas son creíbles. Existe una puerta, Srta. Spencer, en el ático de la casa de mi padre; un lugar al que sólo acostumbramos a venir los sirvientes y yo, si alguien abriera esta puerta, cosa imposible, se encontraría ante el vacío de cuatro pisos de altura, puesto que más allá no hay estancia. Es una puerta que no va a ninguna parte... Nadie puede cruzarla por mucho que lo intente... pero eso ha cambiado, ya que usted, Srta. Spencer, me ha abierto esa puerta. Usted es la llave. [...]

[Amanda] Vale. Elizabeth Bennet está mi cuarto de baño. Evidentemente veo visiones ¿Por qué? ¿Habré leído demasiado a Austen? (Cap.1, 00:03:54 - 00:05:11; énfasis añadido).

\section{Gráfico no 1: Elizabeth Bennet en el baño de Amanda Price}

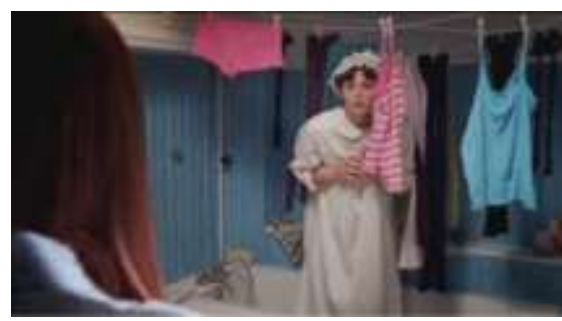

Fuente: Persiguiendo a Jane Austen, Dan Zeff, 2008

En este primer contacto entre las dos protagonistas, obtenemos la clave de por qué se abre una puerta entre el mundo de Amanda y el de Elizabeth. Es el deseo y la necesidad de Amanda lo que hace que esa puerta se abra, ella es la llave (énfasis añadido). Después de que su novio le haya pedido matrimonio, Amanda piensa en lo poco romántico que ha sido (eructa antes de decirle "¡Vamos! Cásate conmigo") deseando que su comportamiento y sus maneras de 
pedir matrimonio fuesen igual a las formas de la época de Austen. Y en esta situación es cuando aparece Elizabeth por primera vez. Sin embargo, Amanda cree que son imaginaciones suyas pues lleva todo el día leyendo Orgullo y Prejuicio. El segundo anacronismo seleccionado es el más significativo de todos, pues es donde se produce el intercambio cultural e histórico en la historia: Amanda, fascinada con el descubrimiento de la puerta que conecta las dos casas se asoma a mirar pero se queda encerrada en 1813 mientras que Elizabeth permanece en 2008 (Cap.1, 00:09:07 - 00:09:41). Para Kaplan, este intercambio suscribe la incorporación de un nuevo personaje en la trama principal de la ficción produciéndose un caos cultural y metaficcional (2008:242-243). Es importante observar que en su segunda visita, Elizabeth aparece vestida con ropa de calle y no con el pijama como la vez anterior, lo que nos induce a pensar que ya viene preparada, si no para quedarse, sí para investigar ese nuevo mundo que le es desconocido y que tanto le fascina.

A partir de aquí, muchos de los anacronismos que se suceden se producen por el mero hecho de que Amanda proviene de una época posterior a la de la historia de Austen. En varias oca- siones se suceden cuando Amanda se siente insegura en un entorno social que, a pesar de que conoce de memoria desde hace catorce años, se siente ignorante por no saber cómo funcionan las cosas. No sólo es Amanda el elemento anacrónico de esta historia, sino que, desde su llegada a Longbourn, los caracteres de los personajes ficticios también cambian y evolucionan pues utilizan su mente y su cuerpo, dejando al margen los pensamientos y los actos con los que Austen les creó.

Cuando Amanda llega a Longbourn se lleva consigo el libro de Orgullo y Prejuicio; aunque no lo lea durante su estancia, el libro está presente en esta época, en la cual que fue concebido, siendo un elemento discordante con este tiempo histórico. En el episodio tres, Amanda lo rompe porque Darcy le dice que no puede casarse con ella porque "no es doncella" (Cap.3, 00:38:44), sin embargo, no consigue que desaparezca sino que acaba en una fuente del jardín de Pemberley a la vista de cualquier persona, siendo Darcy al que descubrimos leyéndolo (Cap.3, 00:43:32).

Después de que el Sr. Bennet se haya golpeado la cabeza, Amanda regresa desesperadamente a Londres en busca de Elizabeth, pero no viaja sola, sino que Darcy la sigue apareciendo también en el Londres de Amanda; en este 
momento, él es anacrónico en la época contemporánea, sobre todo, cuando le vemos en el autobús, reconociendo a "Tinky Winky"x, observando la televisión, la plancha o el ordenador. Además, el hecho de que reconozca, por segunda vez y en el Londres actual, que ama a Amanda, contribuye, una vez más, a demostrar la cantidad de anacronismos que aparecen en la serie, aportando la diferencia cultural y temporal que se quiere demostrar con la inclusión de todos estos elementos en la historia.

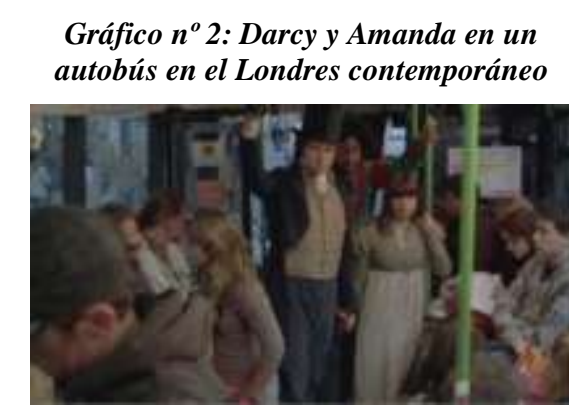

Fuente: Persiguiendo a Jane Austen, Dan Zeff, 2008

En el momento en que encuentran a Elizabeth, Amanda se da cuenta del cambio que ha dado, pues se ha adaptado perfectamente a la época en la que vive: viste moderna, tiene móvil, ordenador, sabe navegar por Internet, sabe la historia de amor con Darcy, es macrobiótica y utiliza la tarjeta de crédito para pagar un taxi. $\mathrm{Al}$ igual que Amanda nos quedamos sorprendidos cuando afirma que: "Nací en la época equivocada, Srta. Price. Fuera de tiempo y fuera de lugar" (Cap.4, 00:23:21).

Kaplan (2010) asegura que los anacronismos que se suceden en esta serie son aceptados por los espectadores como "bromas ingeniosas", que, ni se los cuestionan, ni les preocupan, pues los aceptan como elementos esenciales para reconocer esa diferencia cultural que existe entre principios del siglo XIX y el siglo XXI. Así, el que ofrezcan chocolate a Amanda para desayunar en Longbourn (Cap.1, 00:17:03), que Elizabeth diga "lencería" (Cap.1, 00:04:36) o que Michael (el novio de Amanda) diga que Elizabeth "limpia los dientes de los niños con tiza" (Cap.4, 00:16:58) son acciones que ayudan a comprender el desconcierto que ciertas situaciones le provocan a Amanda.

\subsection{Influencias contemporáneas en la reinterpretación de la historia de Orgullo y Prejuicio}

Persiguiendo a Jane Austen es una historia alocada, irónica e irreal que reinterpreta la novela de Austen a los valores contemporáneos en los que la postmodernidad está presente; de esta manera comprobamos que, a lo largo de la serie, se producen situaciones que nada tienen que ver con el origi- 
nal, pues busca el entretenimiento sobre la fidelidad.

Son muchos los elementos, tanto expresiones verbales como objetos, que Amanda incorpora a su nueva vida en la era de la Regencia y que influirán en el desarrollo posterior de las vidas de estos personajes. Desde el momento que Amanda aparece en Longbourn ella viene cargada con un bagaje cultural contemporáneo: lleva ropa moderna (chaqueta de cuero negra, blusa morada, vaqueros apretados, cinturón a la cintura, botas rojas), pelo pelirrojo, teléfono móvil, lápiz labial y cigarros, que la ponen fuera de lugar tanto en la biblioteca con el Sr. Bennet como desayunando con las Srtas. Bennet, por lo que tiene que justificar inmediatamente su indumentaria alegando que es para cazar nutrias. Sin embargo, los artefactos culturales como los cigarros o el lápiz labial se entremezclan con las casas majestuosas, los rituales de cortesía y de noviazgo y, sobre todo, con los trajes de la época, proporcionando el contraste cómico dentro del complot clásico de la novela de Austen.

No sólo la ropa y los objetos que ella incorpora al mundo de Austen influyen en los comportamientos y acciones de los demás personajes, sino que con sus expresiones un tanto vulgares para aquella época la hacen todavía más diferente e influyente. Así, en su estancia en Longbourn, cuando Lydia se mete en la cama con ella, Amanda enseguida cree que se trata de un reality show o de una película como la de El show de Truman (1998). Pero no son las únicas, pues a lo largo del serial, Amanda introduce palabras contemporáneas y ajenas a la vida diaria de las hermanas Bennet: sándwich, lentillas, gripe, paracetamol, neón, jumbo (tipo de avión) o ¡Petarda! como un juego de cartas. Todas estas expresiones sorprenden a los personajes decimonónicos $\mathrm{y}$, en ocasiones, estas palabras son tomadas por otros personajes, como es el caso de Lydia que repite "suficiente para aparcar un maldito jumbo" (Cap.2, 00:29:36). Las referencias contemporáneas en el capítulo tres son menos frecuentes porque Amanda ya se ha adaptado a la época decimonónica y evita, en la medida de lo posible, decir palabras que les resulten extrañas a sus ahora coetáneos. Para Laurie Kaplan, todas estas expresiones verbales de Amanda la otorgan los instrumentos verbales necesarios para minar a Caroline Bingley, a la Sra. Catherine de Bourgh y a la Sra. Bennet, además

she acts out against the horrid $\mathrm{Mr}$. Collins; she rebukes Mr. Bingley when he gives up his pursuit of Jane, asserting that "it was badly done," and she will not allow his 
excuse that his friend Darcy is too strong for him. Frustrated, Amanda blurts out for him a description of Mr. Collins's "hands slithering all over" Jane. It is a gross image, but Amanda says aloud what many readers have been thinking about poor Charlotte for two hundred years. (2008:251)

En el último episodio no es sólo Amanda la que introduce expresiones propias de la cultura contemporánea, sino que Elizabeth tras su regreso a Longbourn viene con las mismas referencias culturales que Amanda, pues ha vivido durante un tiempo en el Londres contemporáneo. Tiene un gran conocimiento sobre la novela de Austen lo que le permite recriminar a su hermana por haberse casado con el Sr. Collins (Cap.4, 00:28:15 00:28:27), e incluso, trae consigo una botellita de alcohol o agua oxigenada con la que cura a su padre (Cap.4, 00:29:26 - 00:29:35).

No son sólo las referencias verbales las que determinan el juego entre lo contemporáneo y lo decimonónico, sino que las alusiones subliminales a otras novelas de Austen y adaptaciones de su novela, Orgullo y Prejuicio, contribuyen a fomentar y apoyar la creación postmoderna de esta versión. En cuanto a la influencia de otras novelas, el nombre de Amanda Price se asocia al de la protagonista de Mansfield Park, Fanny Price. Su carácter es sensible, romántico, apasionado y soñador como el de Marianne Dashwood de Sentido y Sensibilidad. Con respecto a otras adaptaciones de Orgullo y Prejuicio, la escena que más destaca es aquella en que Amanda le pide a Darcy como favor que se introduzca en el lago que tiene en su jardín. Esta escena alude a la famosa zambullida de Darcy/Firth en la versión de 1995. Para Kaplan (2010), Persiguiendo a Jane Austen desmonta momentos icónicos que la industria cinematográfica de herencia ha hecho irresistibles, como es el caso de esta escena. A diferencia de la versión anterior, en esta serie se ha transformado, pues es Amanda quien le induce a hacerlo como un favor personal para comprobar que el efecto que le produjo cuando lo vio en la serie de la BBC fue el mismo.

Las otras referencias que se incluyen tienen relación con la adaptación que hizo Joe Wright en 2005. Se nombra en siete ocasiones a "Lady Ambrosia" o Ambrosia. Al principio, pensamos que es una criada, pero pronto Charlotte nos saca de nuestra ignorancia y descubrimos quién es:

[Charlotte] Es una excentricidad muy característica de nuestra amiga a ella se lo cuenta... pero... aún más extraño que Ambrosia se lo 
haya contado a usted... porque al fin y al cabo... Lady Ambrosia no deja de ser una cerda de granja. (Cap.1, 00:26:24 - 00:26:35)

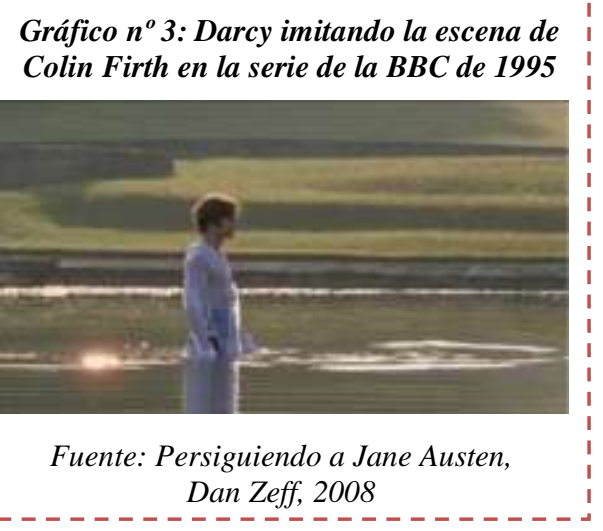

Este cerdo hace referencia, aunque no coincidan en el sexo, al que aparece en la versión de 2005, en el minuto 00:18:10 - 00:18:17. La otra alusión a esta versión es la continua aparición de gallinas, patos y animales de granja que aparecen tanto en la casa de los
Bennet (Cap.1, 00:15:19) como en Meryton (Cap.3, 00:04:31) que al igual que la versión de Wright aparecen en estos escenarios (00:01:36 / 01:34:12).

Para Kaplan, Elliot Cowan, el actor que encarna a Darcy, es una mezcla de los tres actores de las tres adaptaciones clásicas que se han hecho de Orgullo y Prejuicio: Matthew Macfadyen de 2005, Colin Firth de 1995 y David Rintoul de 1980 (2008:246).

Janet Todd y Linda Bree elogian la serie por ser, principalmente, una adaptación diferente que usa la novela como un punto de partida pero que crea algo original, distinto a una adaptación fiel (Kaplan, 2010). El envío de una protagonista moderna al mundo de la Regencia se debe, tanto a la gran influencia que inspira la industria de Jane Austen, como a la interacción que surge entre la obra de la autora con otros textos contemporáneos.

\section{Conclusiones}

La historia que aparece en Orgullo y Prejuicio es fácilmente adaptable a otras épocas, pues la trama es similar a las comedias románticas que existen en la actualidad, en las que una chica conoce a un chico del que se enamora, al que enseguida odia, para después reenamorarse y casarse con él. Nos apoyamos en las palabras de Casilda de
Miguel y otros cuando aseguran que, aunque sean películas protagonizadas por mujeres y traten de romper con el poder patriarcal que fue incorporado al ámbito cinematográfico hace muchos años, éstas no terminan de reflejar los cambios que se han producido a lo largo de la historia del feminismo. Para estas autoras, la imagen de la 
nueva mujer apenas ha cambiado y dista poco de los estereotipos conservadores "que explotan la dimensión tradicional de lo "femenino"" (De Miguel, Olabarri e Ituarte, 2004:95-97). Sin embargo, se observa que a través de esta adaptación postmoderna se trasladan los componentes feministas existentes en la novela a la época actual, donde se adecúan mejor tanto en los personajes como en sus comportamientos. La protagonista rompe con los valores establecidos, buscan el triunfo profesional, decide por propio derecho y rompe las barreras sociales y laborales para lograr la igualdad y la libertad femenina.

Persiguiendo a Jane Austen pertenece al grupo de adaptaciones postmodernas que se hacen de la novela, pero en ella se observa la esencia de la novela de Austen y la influencia de las versiones contemporáneas que se han hecho de Orgullo y Prejuicio aportando un nuevo significado y, sobre todo, una nueva manera de abordar las adaptaciones de las novelas, a las que se les confieren valores totalmente contemporáneos que reivindican la evolución cultural a la vez que se reclaman ciertos valores tradicionales que nos permitirían llevar una vida mejor. Esta adaptación, es la versión que presenta un carácter más marcadamente postmoderno, pues no se toma demasiado en serio la narración original y a través del humor, la parodia y el absurdo da una vuelta de tuerca a la novela de Austen, a la que no trata de venerar, sino de reelaborar en un contexto diferente y un tanto extravagante, como es la intrusión de un personaje contemporáneo en la época de la Regencia. Para ello, como he señalado, utiliza la cita, la intertextualidad, los anacronismos y la invención y alteración de acciones y personajes. Sería curioso abordar esta adaptación desde un punto de vista feminista para comprobar cómo influyen los postulados femeninos como la independencia femenina, la libertad sexual o la educación de un personaje contemporáneo como es Amanda Price en su interacción con los personajes femeninos decimonónicos sumidos en la obediencia y en el poder masculino. 


\section{Referencias}

Assiter, A. (1996). Enlightened Women. Modernist Feminism in a Postmodern Age. Londres: Ed. Routledge.

Butler, A.M. (2005). Films Studies. Vermont: Pocket Essentials.

Callinicos, A. (1993). Contra el

Postmodernismo. Colombia: Ed. Áncora.

De Miguel, C., Olabarri, E. e Ituarte, L. (2004). La identidad de género en la imagen fílmica. Bilbao: Universidad del País Vasco.

Dempster, S. (04/09/2008). Lost in Lost in Austen: Episode 1 en The Guardian, (http://www.guardian.co.uk/culture/tvandradio blog/2008/sep/04/lostinlostinaustenep1?INTC MP=SRCH) (Fecha de consulta: 20/12/2011).

Garrett, R. (1995). Costume Drama and Counter Memory: Sally Potter's Orlando en Dowson, J. y Earnshaw, S. (Eds.). Postmodern Subjects/Postmodern Texts. Amsterdam: Ed. Rodopi B. V..

Gibson, J. (15/09/2008). Creative revival is not enough to reserve ITV's historic low en The

Guardian,(http://www.guardian.co.uk/media/2 008/sep/15/itv.television) (Fecha de consulta: 20/12/2011).

Heller, Á. y Fehér, F. (1989). Políticas de la Postmodernidad. Ensayos de Crítica Cultural. Barcelona: Ed. Península.

Jameson, F. (1991). Ensayos sobre el postmodernismo. Compilado por Horacio Tarcus.

Buenos Aires: Ed. Imago Mundi.

Kaplan, L. (2008). 'Completely without sense': Lost in Austen en Persuasions: The Jane Austen Journal no 30, pp. 241-254. . (2010). Lost in Austen and Generation-Y Janeites en Persuasions: The Jane Austen Journal On Line vol. 30, $n^{\circ} 2$.
Lyotard, J.F. (1987). La Condición Postmoderna. Madrid: Ed. Cátedra.

Modleski, T. (2008). Loving with a Vengeance. Mass-produced Fantasies for Women. Nueva York: Ed. Routledge.

Pérez Ríu, C. (2000). La mujer victoriana en novelas inglesas contemporáneas y sus adaptaciones cinematográficas. Oviedo: Servicio de Publicaciones Universidad de Oviedo.

Teeman, T. (04/09/2008). God on Trial; Lost in Austen: Last's Night's TV en The Times, (http://entertainment.timesonline.co.uk/tol/arts and_entertainment/tv_and_radio/article46680 35.ece) (Fecha de consulta: 16/03/2011).

Wellmer, A. (1993). Sobre la dialéctica de modernidad y postmodernidad. La crítica de la razón después de Adorno. Madrid: Visor Distribuciones.

Wilkes, N. (25/09/2008). 'Lost in Austen' ends with $3 \mathrm{~m}$ en Digital

Spy, (http://www.digitalspy.co. uk/tv/news/a131078/lost-in-austen-ends-with3m.html) (Fecha de consulta: 20/12/2011).

Zeff, D. (2008). Persiguiendo a Jane Austen. (Lost in Austen). [Serie]. Reino Unido: ITV / Mammoth Screen

Cita de este artículo

Romero González, I. (2012) La influencia de la Postmodernidad en Orgullo y Prejuicio (1813): Persiguiendo a Jane Austen (2008), Dan Zeff. Icono14 10(2), 6-28, doi: $\underline{10.7195 / \text { ri14.v10i2.150 }}$ 


\section{NOTAS}

${ }^{\mathrm{i}}$ Las adaptaciones teatrales que se han realizado sobre esta novela son: Pride and Prejudice de Helen Jerome (1935); First Impressions, musical de Broadway (1959); Pride and Prejudice de Bernard J. Taylor (1995); y, Pride and Prejudice de Jon Jory (2006).

ii Estas referencias se observan en series animadas como Futurama o Wishbone (http://janeausten.zxq.net/ mixpelis.htm) (Fecha de consulta: 16/02/2011).

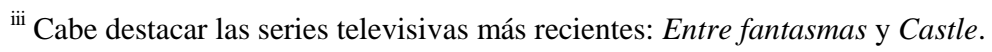

${ }^{\text {iv }}$ Un ejemplo de ello es La casa del lago (2006), protagonizada por Sandra Bullock y Keanu Reeves.

${ }^{v}$ Varios escritores como Pamela Aidan, Elizabeth Aston, Amanda Grange o Emma Tennat, entre otros muchos, han escrito libros sobre Elizabeth y Darcy para continuar la historia que escribió Jane Austen en 1813. Una de estas novelas es Orgullo y Prejuicio y Zombies, escrita por Seth GrahameSmith en 2009 y que ha tenido gran éxito entre los lectores americanos. Las productoras que está adaptando esta novela al cine son Handsomecharlie Films, Darko Entertainment y Lionsgate. P.D James, la célebre escritora de novelas policíacas, ha publicado en el mes de noviembre una secuela de Orgullo y Prejuicio, titulada Death comes to Pemberley, en la que retoma la historia de Austen seis años después de que los protagonistas se hayan casado y en la que están inmersos en una trama de asesinato.

${ }^{v i}$ Pertenecen a esta categoría de adaptaciones postmodernas, El diario de Bridget Jones (2001), Orgullo y Prejuicio: la película (2003), Bodas y Prejuicios (2004) y Persiguiendo a Jane Austen (2008), objetos de estudio del presente artículo.

${ }^{\text {vii Lucía Solaz, "El cine postmoderno" (http://www.encadenados.org/n39/cine postmoderno.htm) }}$ (Fecha de consulta: 15/12/2011).

viii Lucía Solaz especifica que el problema del nuevo movimiento cultural se refiere más a la actitud que a la simple mezcla de elementos de origen diverso, pues trata de reivindicar el "individualismo, lo plural, lo híbrido, lo ambiguo y lo complejo, frente a lo único, lo puro, lo claro y lo sencillo". (http://www.encadenados.org/n39/cine_postmoderno.htm) (Fecha de consulta: 15/12/2011).

${ }^{\text {ix }}$ Los Janeites son los devotos de las obras de Jane Austen que idolatran todo lo que tiene relación con ella como son las adaptaciones, el merchandising, la época o el vestuario.

${ }^{\mathrm{x}}$ Tinky Winky es un personaje de la serie infantil Teletubbies que fue un programa de televisión creado en 1997 por la cadena británica BBC, con contenidos orientados a bebés y a niños de edad preescolar. 